\title{
Transverse Dynamics at RHIC
}

$\mathrm{Nu} \mathrm{Xu}$ Lawrence Berkeley National Laboratory, Berkeley, CA 94720, USA

Zhangbu Xu Brookhaven National Laboratory, Upton, NY 11794, USA

\section{Initial Conditions}

Partonic collectivity refers to collective motion, i.e., hydrodynamic type of flow, involving partonic degrees of freedom. Large initial energy deposition at central rapidity and subsequent strong interaction at the partonic level is an important initial condition for partonic collectivity. We first discuss what the measured average transverse momentum distribution $\left\langle p_{t}\right\rangle$ is. The left panel of Fig. 1 shows the $\left\langle p_{t}\right\rangle$ of negatively charged particles $\left(h^{-}\right)$within $|\eta|<0.5$ or total charged particles $\left(N_{c h}\right)$ as a function of $\sqrt{s}$ for $p p, \bar{p} p$ (open circles), $e^{+} e^{-}$(open triangles), and $A A$ (filled circles) collisions [1,2]. For the $p p$ collisions the dashed-line is the parameterization of the measured mean $p_{t}$ [3]. For the $e^{+} e^{-}$data, the straight dashed-line is an approximation of JETSET calculations [4]. The fact that the $\left\langle p_{t}\right\rangle$ from $A A$ collisions is distinctly different from both $p p$ and $e^{+} e^{-}$indicates that the $A A$ collisions are not simple superpositions of the elementary collisions. In heavy ion collisions, the increase of $\left\langle p_{t}\right\rangle$ as a function of beam energy or centrality is much larger than expected from the soft-hard two-component model [5]. For example, the $\left\langle p_{t}\right\rangle$ difference between SPS and RHIC is a factor of 5 larger than the $\left\langle p_{t}\right\rangle$ difference between these two energies in $p p$ collisions. In a simple two-component model, the maximum difference is a factor of 3 if the increase in $\left\langle p_{t}\right\rangle$ is solely due to the increasing contribution from the hard scattering component. The right panel of Fig. 1 also shows the calculation from RQMD with and without rescattering and HIJING which underpredicts the $\left\langle p_{t}\right\rangle$.

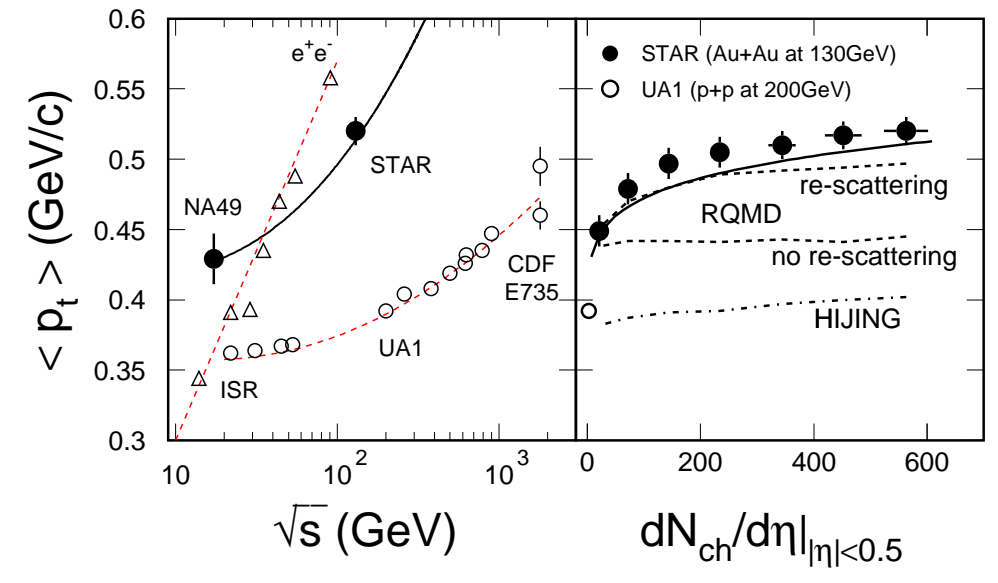

Figure 1 (Left): Negatively charged hadron $\left\langle p_{t}\right\rangle$ as a function of beam energy $\sqrt{s}$ for $e^{+} e^{-}, p p, \bar{p} p$ (open symbols) and central $A A$ (filled symbols) collisions. (Right): $\left\langle p_{t}\right\rangle$ as a function of collision centrality. The solid-curves represent $\left\langle p_{t}\right\rangle$ scaling and the dashed ones represent RQMD with/without rescatterings and the default HIJING(1.35) results.

Both saturation [6] and hydrodynamic [7] models predict some scaling behavior of $\left\langle p_{t}\right\rangle$ $\sim \sqrt{(d N / d \eta)_{A A} / \pi R^{2}}$ when the conditions are satisfied. The solid curves in Fig. 1 show the energy dependence and centrality dependence of $\left\langle p_{t}\right\rangle$ as discussed in Ref. [8]. However, 
the preliminary STAR data [9] show that the increase in $\left\langle p_{t}\right\rangle$ from $\sqrt{s_{\mathrm{NN}}}=130$ to 200 $\mathrm{GeV}$ is not as strong as the thick-line indicates which may invalidate the scaling law from the saturation model [6]. On the other hand, the energy dependence might indicate the importance of early thermalization in the partonic stage, as proposed by A. Mueller et al. [10]. Such initial partonic activity is consistent with the early development of flow as indicated from $\left\langle v_{2}\right\rangle$ measurements at RHIC [11]. It will be interesting to see if the description holds with an energy scan at lower beam energies at RHIC.

\section{Partonic Collectivity at RHIC}

The transverse momentum distributions [12,13], from central $\mathrm{Au}+\mathrm{Au}$ collisions at $\sqrt{s_{\mathrm{NN}}}=130 \mathrm{GeV}$, are shown in Figure 2. One can see that at $p_{t} \sim 2 \mathrm{GeV} / \mathrm{c}$, all heavier mass particle yields approach that of lighter ones. This behavior does not depend on the particle type except their mass. The mass dependence indicates a strong transverse collective expansion in the collisions at RHIC. More importantly, from the azimuthal anisotropy $\left\langle v_{2}\right\rangle$ [11] combined with the spectra results, one might conclude that the collective expansion has already developed at the early partonic stage.

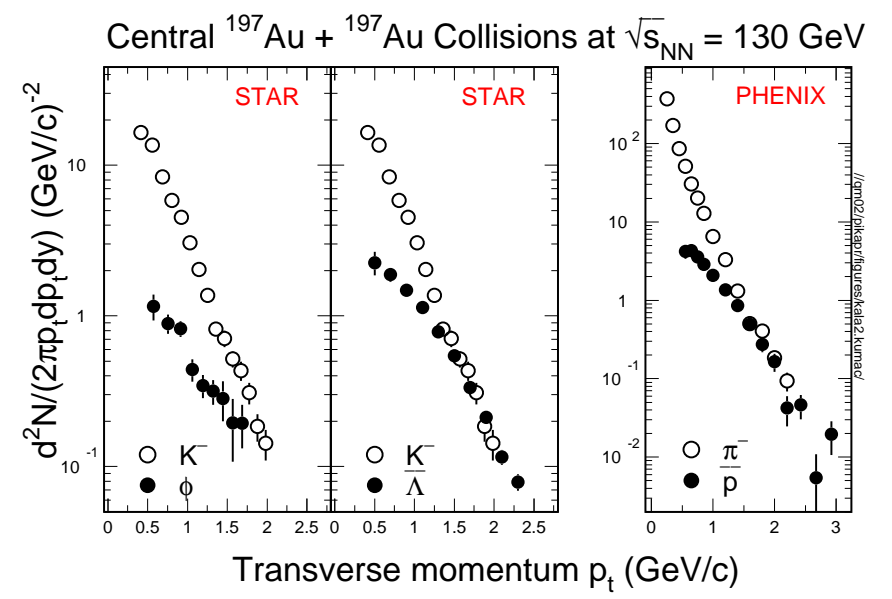

Figure 2 Transverse momentum spectra from central $\mathrm{Au}+\mathrm{Au}$ collisions at $\mathrm{RHIC}\left(\sqrt{s_{\mathrm{NN}}}=130 \mathrm{GeV}\right)$. At $p_{t} \sim 2 \mathrm{GeV} / \mathrm{c}$, heavier particle yields are approaching that of lighter ones - indicating a strong collective expansion developed in those collisions.

The measured transverse momentum distributions also have been fitted by the function $f=A \cdot \exp \left(-m_{t} / T\right)$, where $m_{t}=\sqrt{p_{t}^{2}+m^{2}}, T$ is the inverse slope parameter, and $A$ is a normalization constant. The magnitude of the slope parameter provides information about the temperature (random motion in local rest frame) and collective transverse flow. Fig. 3(left) shows the measured particle slope parameters from $\mathrm{Pb}+\mathrm{Pb}$ central collisions at $\sqrt{s_{\mathrm{NN}}}=17.2 \mathrm{GeV}[14-17]$ (open symbols) and from $\mathrm{Au}+\mathrm{Au}$ central collisions at $\sqrt{s_{\mathrm{NN}}}$ $=130 \mathrm{GeV}[12,13,18]$ (filled symbols). As one can see in the figure, at the SPS energy, the values of the slope parameters for the $\Omega$ baryon, charm particles $J / \psi$, and $\psi^{\prime}[16,17]$ are all about $240 \mathrm{MeV}$ independent of the mass. The straight hatched band indicates the possible variation of the slope parameters due to initial scatterings [19]. The values of the slope parameters from other light hadrons like $\pi, K$, and $p$, on the other hand, show a linear increase as the mass increases. Such a mass dependence of the slope parameter can be explained by collective flow within the framework of hydrodynamics [14,20-22].

In the hadronic phase, the elastic scattering cross sections for particles like $\phi, \Omega$, and $J / \psi$ are smaller than that of $\pi, K$, and $p[23]$. Therefore, the interactions between them 
and the rest of the system are weak, leading to the flat band behavior in Fig.3(left). On the other hand, the slope parameter of these weakly interacting particles may reflect some characteristics of the system at hadronization. Then it should be sensitive to the strength of the color field [24-26]. Under this assumption, the fact that the weak interacting particles show a flat slope parameter as a function of particle mass would indicate that the flow develops at a later hadronic stage of the collision at SPS.

In figure 3(right panel), the integrated values of $v_{2}$ demonstrate a mass dependence as predicted by hydrodynamic calculations [11,27]. In terms of hydrodynamics this mass dependence is due to the anisotropic pressure gradient pushing massive particles outward in velocity space. The width of the gray-band indicates the uncertainties of the model calculations, mostly due to the choice of the freeze-out conditions [27]. A key element in the calculation is that the system develops the anisotropy at a relatively early stage of the collision when partonic degrees of freedom dominate the interactions. At higher collision energies, the increased contribution from the partonic interactions lead to the observed systematic increase of $v_{2}[11]$.
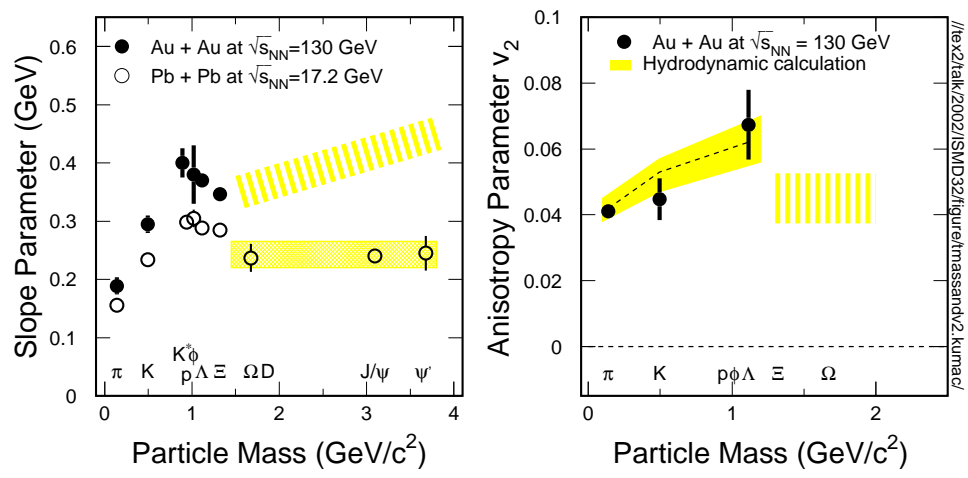

Figure 3 (Left) The slope parameter as a function of particle mass. Open and filled symbols represent the results from $\mathrm{Pb}+\mathrm{Pb}$ and $\mathrm{Au}+\mathrm{Au}$ central collisions at SPS and RHIC, respectively. (Right) $v_{2}$ as a function of particle mass. The gray-band indicates the hydrodynamic model results [27].

At RHIC energies, see filled symbols in Fig.3(left), the mass dependence of the slope parameter seems to be stronger than that at SPS energies, indicating a larger collective flow in higher energy nuclear collisions. The strong energy dependence of the slope parameters might be the consequence of the larger pressure gradients at RHIC energies. With a set of reasonable initial/freeze-out conditions and equation of state, the stronger transverse expansion at RHIC energies was indeed predicted by hydrodynamic calculations [20,27]. Should the collective flow develop at the partonic level, one would expect a mass dependence of the slope parameters for particles like $\Omega, J / \psi$, and $\psi^{\prime}$ as indicated by the dashed band in Figure 3(left) $[21,28,29]$. For the same reason, one would expect a non-zero value of $v_{2}$ for those particles as indicated by the dashed band in the right panel of Fig.3.

\section{Summary}

Studies of $\left\langle p_{t}\right\rangle$, transverse momentum spectra and anisotropy flow from nuclear collisions at RHIC indicate early thermalization and strong collective expansion. We propose a systematic study of the anisotropy parameter $v_{2}$ and the transverse momentum distributions of $\phi, \Omega, D^{0}, \Lambda_{C}$ and $J / \psi$ in order to extract information on partonic collective flow.

We are grateful for many enlightening discussions with Drs. P. Braun-Munzinger, K. 
Haglin, P. Huovinen, D. Kharzeev, H.G. Ritter, J. Schaffner-Bielich, K. Schweda, E.V. Shuryak, R. Snellings, S. Soff, D. Teaney, T. Ullrich, and E. Yamamoto. This work has been supported by the U.S. Department of Energy under Contract No. DE-AC03$76 \mathrm{SF} 00098$.

\section{REFERENCES}

1. C. Adler et al., (STAR Coll.), Phys. Rev. Lett. 87, 112303(2001); ibid. submitted to Phys. Rev. Lett., nucl-ex/0206011.

2. H. Appelshäuser et al., (NA49 Coll.), Phys. Rev. Lett. 82, 2471(1999).

3. C. Albajar et al., (UA1 COll.), Nucl. Phys. B335, 261(1990).

4. R. Akers et al., (OPAL Coll.) Phys. Lett. B320, 417(1994).

5. D. Kharzeev and M. Nardi, Phys. Lett. B507, 121(2001); S.Y. Li and X.N. Wang Phys. Lett. B527, 85(2002).

6. L. McLerran et al., Phys. Lett. B514, 29(2001); J. Schaffner-Bielich et al., nuclth/0202054.

7. D. K. Srivastava, Phys. Rev. C64, 064901(2001).

8. Z. Xu et al., (STAR Coll.), nucl-ex/0207019.

9. G. Van Buren et al., (STAR Coll.), these proceedings.

10. A. Mueller et al., these proceedings; R. Baier et al., Phys. Lett. B539, 46(2002).

11. K.H. Ackermann et al., (STAR Coll.), Phys. Rev. Lett. 86, 402(2001); C. Adler et al. (STAR Coll.), Phys. Rev. Lett. 87, 112301(2001); ibid. 89, 132301(2002).

12. C. Adler et al. (STAR Coll.), Phys. Rev. Lett. 87, 262302(2001); ibid. 89, 092301(2002); ibid. Phys. Rev. C65, 04901(2002); ibid. submitted to Phys. Lett. B, nucl-ex/0206008; ibid. submitted to Phys. Rev. Lett., nucl-ex/0205015.

13. K. Adcox et al., (PHENIX Coll.), Phys. Rev. Lett. 89, 092302(2002); ibid. 88, 242301(2002).

14. I. G. Bearden et al., (NA44 Coll.), Phys. Rev. Lett 78, 2080(1997).

15. E. Andersen et al., (WA97 Coll.), Phys. Lett. B433, 209(1998).

16. P. Bordalo et al., (NA50 Coll.), Nucl. Phys. A698, 127c(2002).

17. M.C. Abreu et al., (NA50 Coll.), Phys. Lett. B499, 85(2001).

18. Mauel Calderón de la Sánchez et al., (STAR Coll.), Nucl. Phys. A698, 503c(2002).

19. J. Hüfner et al, Phys. Lett. B215, 218(1988).

20. D. Teaney, J. Lauret, and E. Shuryak, nucl-th/0110037.

21. N. Xu and M. Kaneta, Nucl. Phys. A698, 306c(2002).

22. E. Schnedermann, J. Sollfrank, and U. Heinz, Phys. Rev. C48, 2462(1993).

23. H. van Hecke, H. Sorge, and N. Xu, Phys. Rev. Lett. 81, 5764(1998).

24. J. Schwinger, Phys. Rev. 82, 664(1951).

25. S. Soff, et al., Phys. Lett. B471, 89(1999).

26. M. Bleicher, et al., Phys. Rev. C62, R1901(2000).

27. P. Huovinen et al., Phys. Lett. B503, 58(2001).

28. P. Braun-Munzinger and J. Stachel, Nucl. Phys. A690, 119(2001); ibid. Phys. Lett. B490, 196(2000).

29. R. Thews, M. Schroedter and J. Rafelski, Phys. Rev. C63, 054905(2001). 